Article

\title{
Flaring $\gamma$-Ray Emission from High Redshift Blazars
}

\author{
Monica Orienti ${ }^{1}$ * , Filippo D'Ammando ${ }^{1,2}$, Marcello Giroletti ${ }^{1}$, Justin Finke ${ }^{3}$ and \\ Daniele Dallacasa ${ }^{1,2}$
}

1 INAF - Istituto di Radioastronomia, Via Gobetti 101, Bologna I-40129, Italy; dammando@ira.inaf.it (F.D.); giroletti@ira.inaf.it (M.G.); ddallaca@ira.inaf.it (D.D.)

2 Dipartimento di Fisica e Astronomia, Universitá degli Studi di Bologna, Viale Berti Pichat, 6/2, Bologna I-40127, Italy

3 U.S. Naval Research Laboratory, Code 7653, 4555 Overlook Ave SW, Washington, DC 20375-5352, USA; justin.finke@nrl.navy.mil

* Correspondence: orienti@ira.inaf.it

Academic Editors: Jose L. Gómez, Alan P. Marscher and Svetlana G. Jorstad Received: 14 July 2016; Accepted: 16 August 2016; Published: 6 September 2016

\begin{abstract}
High redshift blazars are among the most powerful objects in the Universe. Although they represent a significant fraction of the extragalactic hard X-ray sky, they are not commonly detected in $\gamma$-rays. High redshift $(z>2)$ objects represent $<10$ per cent of the active galactic nuclei (AGN) population observed by Fermi so far, and $\gamma$-ray flaring activity from these sources is even more uncommon. The characterization of the radio-to- $\gamma$-ray properties of high redshift blazars represents a powerful tool for the study of the energetics of such extreme objects and the Extragalactic Background Light. This contribution will present results of multi-band campaigns, from radio to $\gamma$-rays, on PKS 0836+710, PKS 2149-306, and TXS 0536+145. The latter is the highest redshift detection of a flaring $\gamma$-ray blazar so far. At the peaks of their respective flares these sources reached an apparent isotropic gamma-ray luminosity of about $10^{50} \mathrm{erg} \cdot \mathrm{s}^{-1}$, which is comparable with the luminosity observed from the most powerful blazars. The physical properties derived from the multi-wavelength observations of these sources are then compared with those shown by the high redshift population.
\end{abstract}

Keywords: galaxies: active; gamma-rays: general; radiation mechanisms: non-thermal

\section{Introduction}

Blazars are radio-loud active galactic nuclei (AGN), with powerful relativistic jets observed at a small viewing angle. For this reason their emission is strongly enhanced due to Doppler boosting effects and they are expected to be detected up to high redshift.

High redshift $\gamma$-ray blazars are among the most luminous individual objects in the Universe. However, they are under-represented in $\gamma$-ray catalogues. In the Third Fermi Large Area Telescope (LAT) source catalogue (3FGL , [1]) there are 64 objects ( 3.7 per cent of the $\gamma$-ray sources associated with AGN) at $z>2$, and only two at $z>3$ : PKS 0537-286 at $z=3.104$ and TXS 0800+618 at $z=3.033$. In contrast, 10 blazars at redshift $z>3$ were detected in hard X-rays by Swift-BAT [2], INTEGRAL-IBIS [3], and NuSTAR [4,5]. Hard X-ray observations seem more suitable for detecting sources at $z>3$, and this is likely due to a spectral bias. In fact, the inverse Compton peak of high-z objects is shifted towards lower energies as the bolometric luminosity increases [6]. Only 10 objects with $z>2$ are in both the 3FGL and the 70-month BAT catalog [7]. These 10 objects have a harder $X$-ray photon index in the $14-195 \mathrm{keV}$ energy range $\Gamma_{\mathrm{X}}<1.5$ and reach higher apparent isotropic hard $X$-ray luminosity than the BAT sources undetected at $\gamma$-rays (Figure 1, left panel). This supports that the softer X-ray sources are unlikely to be detected in the Fermi LAT energy bands. This is reflected in the presence of only 2 out of these 10 objects in the first Fermi LAT catalog above $10 \mathrm{GeV}$ [8]. 
If we consider the $\gamma$-ray sources at $z>2$ (Figure 1, right panel), the 10 objects detected in both energy ranges have a larger apparent isotropic $\gamma$-ray luminosity in the $0.1-100 \mathrm{GeV}$ energy range $\left(\mathrm{L}_{\gamma}>10^{48} \mathrm{erg} \cdot \mathrm{s}^{-1}\right)$ than those undetected in hard X-rays, while the $\gamma$-ray photon index $\Gamma_{\gamma}$ spans the same interval [7].

High- $z$ objects caught during a $\gamma$-ray flaring episode are even more difficult to find. The flaring threshold commonly used is set to an apparent flux of $10^{-6} \mathrm{ph} \cdot \mathrm{cm}^{-2} \cdot \mathrm{s}^{-1}$ which may be difficult to be reached by distant objects. However, a hardening of the high energy spectrum during a flare may favour the detection. Thirteen FSRQ with $z>2$ have been detected by Fermi-LAT during a $\gamma$-ray flare up to now. In this contribution we present results of a multiwavelength campaign of three high- $z$ objects, TXS 0536+145, PKS 2149-306, and S5 0836+710 detected by Fermi-LAT during a $\gamma$-ray flare. Multi-epoch Swift observations were performed for all of the targets, while a follow-up campaign of radio observations was triggered after the $\gamma$-ray flare of TXS 0536+145 and S5 0836+710.

Throughout this paper, we assume the following cosmology: $H_{0}=71 \mathrm{~km} \mathrm{~s}^{-1} \mathrm{Mpc}^{-1}, \Omega_{\mathrm{M}}=0.27$ and $\Omega_{\Lambda}=0.73$, in a flat Universe.
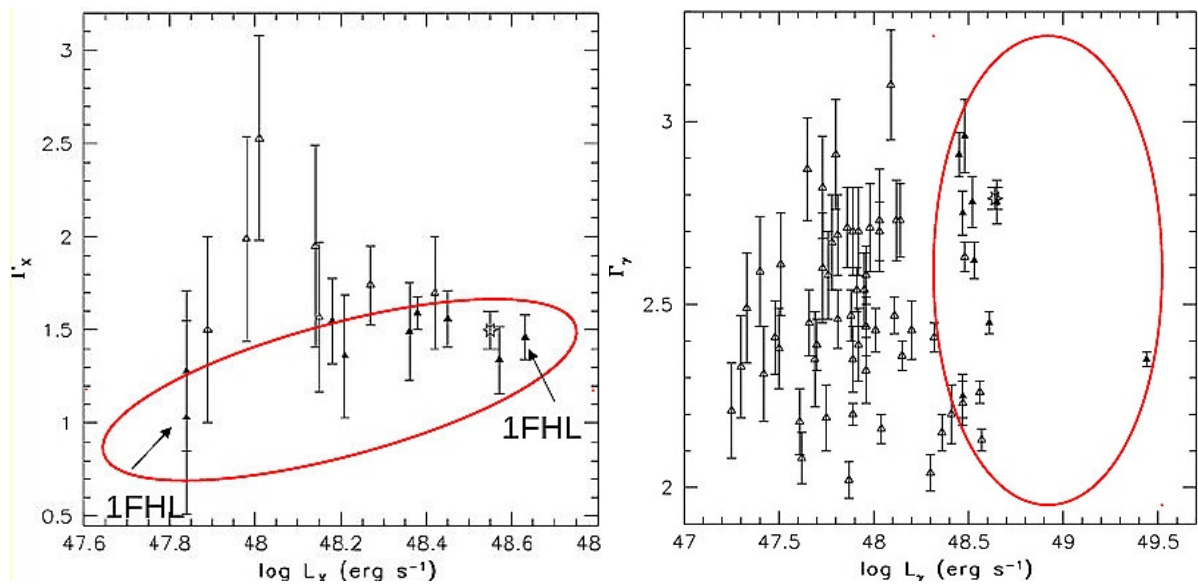

Figure 1. Left panel: X-ray photon index versus X-ray apparent isotropic luminosity in the $14-195 \mathrm{keV}$ energy range for the blazars with $z>2$ detected by Swift-BAT. The filled and open triangles represent the objects detected and not detected by Fermi-Large Area Telescope (LAT), respectively; Right panel: $\gamma$-ray photon index versus apparent isotropic luminosity in the $0.1-100 \mathrm{GeV}$ energy range for the blazars with $z>2$ included in the 3FGL. The filled and open triangles represent the objects detected and not detected by Swift-BAT, respectively. Images adapted from [7].

\section{Results}

In this Section we present results of the $\gamma$-ray, X-ray, and radio observations. The detailed description of the data analysis can be found in $[7,9]$.

\subsection{TXS $0536+145$}

TXS 0536+145 was detected during a very high $\gamma$-ray activity state in 2012 March by Fermi-LAT. With a redshift of $z=2.69$ [10], this source is the $\gamma$-ray flaring blazar at the highest redshift observed so far. The source was not present in the first two Fermi-LAT catalogues [11,12] and no EGRET $\gamma$-ray object was reported at the location of the source. However, on 22 March 2012 the source underwent a major $\gamma$-ray flare reaching an apparent isotropic luminosity of $6.6 \times 10^{49} \mathrm{erg} \cdot \mathrm{s}^{-1}$, i.e., comparable with, or even higher than, the luminosity reached by the majority of bright flaring blazars. During the flare we observed a hardening of the $\gamma$-ray photon index, $\Gamma_{\gamma} \sim 2.0$, with respect to its average value $\left(\Gamma_{\gamma} \sim 2.4\right)$ and the gamma-ray spectrum shows a significant curvature. 
Swift observations performed a few days after the $\gamma$-ray flare pointed out that TXS $0536+145$ was in a relatively bright state also in X-rays, with a flux roughly a factor of 2 higher than the average. No significant spectral change is observed in the X-rays.

Before the 2012 March flare, the source was first detected in $\gamma$-rays on 2012 January showing an enhancement of its high-energy activity, but without reaching a similar peak flux. A tentatively double enhancement of the source activity is visible in the radio light curves constructed making use of Very Long Baseline Array (VLBA), European VLBI Network (EVN), and Medicina single-dish telescope observations. At 15 and $24 \mathrm{GHz}$ the radio maxima are delayed by about 4 months ( $\sim$ in the source frame). At $8.4 \mathrm{GHz}$, the variations are smoother and delayed by $\sim 2$ months with respect to what is observed at higher radio frequencies [9]. This agrees with the presence of opacity effects.

Thanks to the parsec-scale resolution of VLBA/EVN data at three frequencies we investigated changes in the source structure and in the spectral index distribution. The source has a core-jet radio morphology extending up to $\sim 6$ mas $(\sim 48 \mathrm{pc})$ at $8.4 \mathrm{GHz}$ (Figure 2 , left panel). The flux density and spectral variability are localized in the core region, while no significant change is observed in the jet component. This suggests that the region responsible for the the flaring emission is located in the self-absorbed radio core and it is likely responsible for the variability observed from radio to high energies.

\subsection{PKS $2149-306$}

During the first two years of Fermi operation, PKS 2149-306 was observed in a low-activity state, with an average $(0.1-100 \mathrm{GeV})$ flux of $\sim 6.4 \times 10^{-8} \mathrm{ph} \cdot \mathrm{cm}^{-2} \cdot \mathrm{s}^{-1}$ and a photon index $\Gamma_{\gamma} \sim 3.0$ [12]. No significant curvature of the spectrum was reported by [12]. However, if we extend the analysis to the first six years of Fermi observations, i.e., between 2008 August and 2014 August, the $\gamma$-ray spectrum shows significant curvature, well described by a log-parabola model with a spectral slope $\alpha \sim 2.4$, and a curvature parameter around the peak $\beta \sim 0.29$. In this period the source had an average flux of $9.7 \times 10^{-8} \mathrm{ph} \cdot \mathrm{cm}^{-2} \cdot \mathrm{s}^{-1}$.

A strong $\gamma$-ray flare from PKS 2149-306 was detected by Fermi-LAT in 2013 January, reaching a daily peak flux of about $3.0 \times 10^{-6} \mathrm{ph} \cdot \mathrm{cm}^{-2} \cdot \mathrm{s}^{-1}$, i.e., about 50 times higher than the average flux during the low-activity phase. At the redshift of the source, $z=2.345$, this flux corresponds to an apparent isotropic luminosity of about $1.5 \times 10^{50} \mathrm{erg} \cdot \mathrm{s}^{-1}$, comparable to the highest values observed for a blazar so far. During the flare we observed a significant hardening of spectral slope, $\alpha \sim 1.99$, without substantial changes in the curvature, suggesting a shift of the inverse Compton peak to high energies. The analysis of the $\gamma$-ray flare light curve with sub-daily time bins indicates a significant flux variation by a factor of 2 or more on 12-h and 6-h time-scale. In particular, if we assume that the super massive black hole (SMBH) of PKS 2149-306 has a mass of $3.5 \times 10^{9} \mathrm{M}_{\odot}$ [13], the 6-h variability is compatible with the light crossing time of the event horizon of the SMBH [7]. This small size of the emitting region should correspond to a small distance from the central $\mathrm{BH}$, putting the emitting region inside the broad-line region (BLR).

Before the huge flare in 2013 January, PKS 2149-306 underwent a high activity phase at the beginning of 2011 when, on February 18, the flux reached a daily peak of $1.4 \times 10^{-6} \mathrm{ph} \cdot \mathrm{cm}^{-2} \cdot \mathrm{s}^{-1}$, which corresponds to an apparent isotropic $\gamma$-ray luminosity of about $5.3 \times 10^{49} \mathrm{erg} \cdot \mathrm{s}^{-1}$. No significant spectral changes were observed during the 2011 February flare.

\section{3. $550836+710$}

S5 $0836+710$, at a redshift of 2.218 , is characterized by periods of high activity interleaved with quiescent states. This source was detected in $\gamma$-rays by EGRET and showed to be variable at energies above $100 \mathrm{MeV}$ [14]. Although it was not bright enough in the first three months of Fermi normal mission operations to be included in the Fermi-LAT Bright Source List [15], the source is present in the First, Second and Third Fermi-LAT catalogs [1,11,12]. Between 2008 August and 2011 January the source was in a relatively quiescent state, although regularly detected on weekly time scales, 
with an average flux of about $5.2 \times 10^{-8} \mathrm{ph} \cdot \mathrm{cm}^{-2} \cdot \mathrm{s}^{-1}$ and a spectrum well represented by a power law with $\Gamma_{\gamma} \sim 2$.9. In 2011 March, S5 0836+710 entered in a high activity phase with multiple sub-flares, which lasted almost 11 months [16]. During the flaring period the spectrum changed shape deviating from a power law and showing a significant curvature.

At the end of 2015 July the source entered a new high activity period with multiple flaring episodes. The first flare peaked on August 2 when the source reached a daily peak flux of $\sim 5.2 \times 10^{-6} \mathrm{ph} \cdot \mathrm{cm}^{-2} \cdot \mathrm{s}^{-1}$. However it is in 2015 November when the source underwent an extraordinary flare lasting almost two weeks. On November 11 the source reached a daily flux of $\sim 6.2 \times 10^{-6} \mathrm{ph} \cdot \mathrm{cm}^{-2} \cdot \mathrm{s}^{-1}$, that is the highest flux ever observed for this source. Significant flux variation by a factor of 2 or more is clearly visible on sub-day time-scales, with the peak of the second flare resolved with 3-h binning (D'Ammando et al., in preparation). During such a short time scale the source reached an apparent luminosity of about $2.9 \times 10^{50} \mathrm{erg} \cdot \mathrm{s}^{-1}$, higher than the luminosity reached by 3C 454.3 during the outburst in 2010 November [17].

Both $\gamma$-ray flares had a counterpart in X-rays. For the first flare the $\mathrm{X}$-ray peak occurred a couple of weeks before the $\gamma$-ray flare. On the contrary, during the second flare both $X$-rays and $\gamma$-rays reached the peak flux the same day.

The high activity observed by Fermi-LAT triggered a multi-frequency campaign in the radio band. VLBA observations at 15, 22 and $43 \mathrm{GHz}$ were performed almost simultaneously for four epochs separated by about two months. The source has a core-jet structure (Figure 2, right panel). A bright jet component is located at about 3 mas $(\sim 25 \mathrm{pc})$ from the core. The core is variable but the variability amplitude does not exceed 20 per cent, which is much below what is observed in X-rays and $\gamma$-rays. The core spectrum is inverted between 15 and $22 \mathrm{GHz}$ and flattens at higher frequency $\left(\alpha_{r} \sim 0.4\right.$; $\left.S_{v} \propto v^{-\alpha}\right)$. No significant flux density variability is detected in the jet component. Its radio spectrum is steep and stable in all of the epochs. The core is slightly polarized with a fractional polarization of about 0.2 per cent at $15 \mathrm{GHz}$ which increases with frequency. The polarization is higher along the jet, reaching a percentage up to 6 per cent ( $\mathrm{D}^{\prime}$ Ammando et al., in preparation).
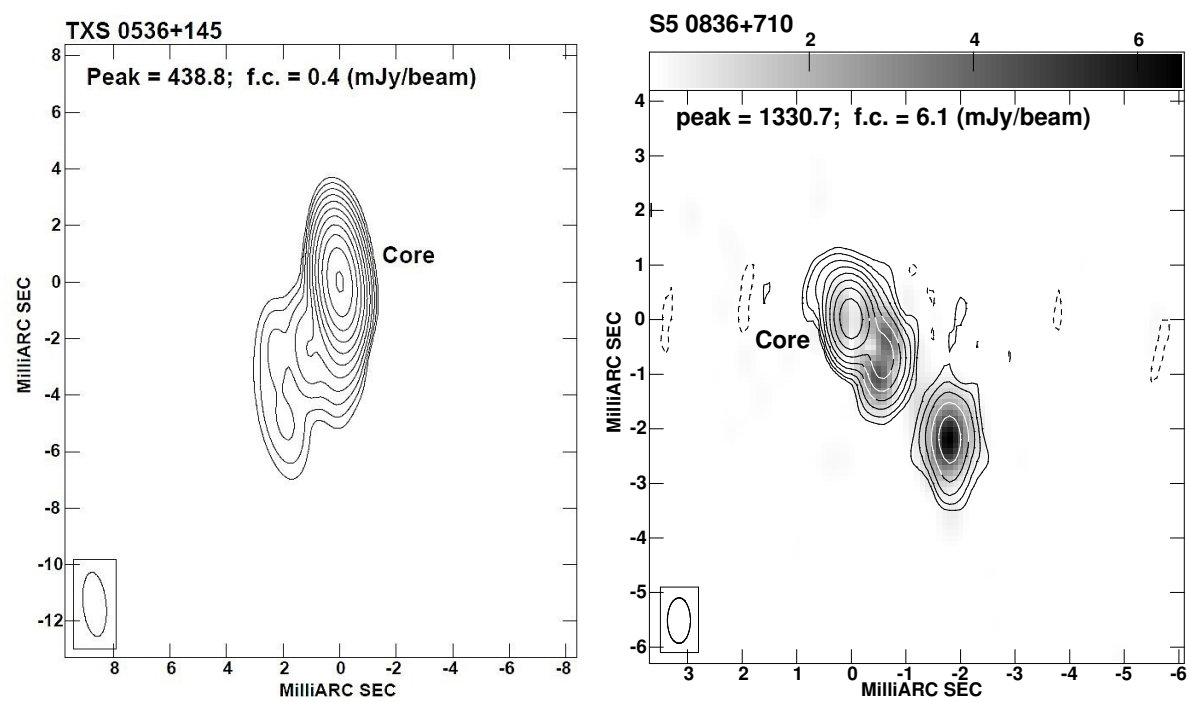

Figure 2. Very Long Baseline Array (VLBA) image at $8.4 \mathrm{GHz}$ of TXS $0536+145$ ( left: the observation was performed in 2012 May) and at $15 \mathrm{GHz}$ of S5 0836+710 (right: the observation was performed in 2016 May). On each image, we provide the peak flux density in $\mathrm{mJy}_{\text {beam }}{ }^{-1}$ and the first contour (f.c.) intensity in $\mathrm{mJy}$ beam ${ }^{-1}$, which corresponds to three times the off-source noise level. Contour levels increase by a factor of 2 . The restoring beam is plotted in the bottom-left corner. The gray scale represents the polarized flux density. 


\section{Discussion}

\subsection{High-Energy Emission}

High-redshift blazars tend to be the most luminous $\gamma$-ray AGN due to their preferential selection by the LAT caused by Malmquist bias [18]. For this reason the objects detected so far represent the tip of the iceberg of the high-z population, while the majority is too faint to be detected individually, while their cumulative emission should contribute to the extragalactic $\gamma$-ray background (see, e.g., [19]).

High-redshift blazars are difficult to detect during an outburst. Only extreme events involving exceptionally high luminosity can be caught by current facilities. Due to their distance the observed flux barely reaches the threshold set for a flare. Among the 64 blazars with $z>2$ in the 3FGL, only 13 were caught during an outburst. The blazars TXS 0536+145, S5 0836+710, and PKS 2149-306 underwent huge $\gamma$-ray flares and their apparent isotropic luminosity increased of almost two orders of magnitude with respect to the average activity state (see Figure 3). During these extraordinary events the apparent luminosity reached $\sim 10^{50} \mathrm{erg} \cdot \mathrm{s}^{-1}$ or more. Such high values were observed only in a handful of objects like 3C $454.3\left(2 \times 10^{50} \mathrm{erg} \cdot \mathrm{s}^{-1}\right.$, [17] $)$ and the other high-redshift gravitationally lensed blazar PKS 1830-211 (2.9 × $\left.10^{50} \mathrm{erg} \cdot \mathrm{s}^{-1},[20]\right)$.

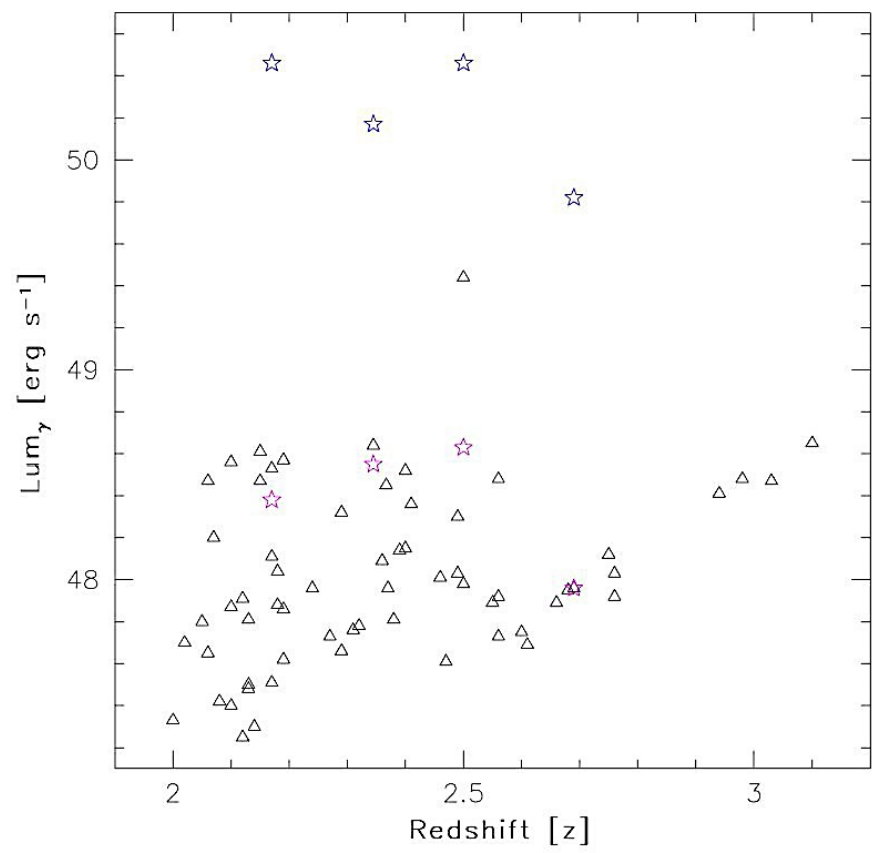

Figure 3. Luminosity versus redshift. Triangles represent the high-redshift sources from the 3FGL with $z>2$ [18], the empty stars represent, from lower to higher $z$, S50836+710, PKS 2149-306, PKS 1830-211, and TXS 0536+145, during the average and flaring activity.

Despite being rare, the study of the high-redshift population is important for our understanding of the extragalactic background light (EBL). This diffuse radiation consists of photons likely produced by stars and galaxies across the Universe and its lifetime. As the high-energy photons from extragalactic sources propagate through the Universe, they interact with EBL photons at ultraviolet through optical wavelengths by $\gamma-\gamma$ absorption, and create electron-positron pairs. This results in an attenuation of the $\gamma$-ray sources above a critical energy that depends on the redshift and the EBL model assumed (see e.g., [21,22]). During the outburst a hardening of the $\gamma$-ray spectrum is usually observed that may extend to higher energies than the range spanned during the non-active state. For the three sources studied here the spectral hardening during the flare is also accompanied by a significant curvature of the spectrum. Considering the EBL model discussed in [22], at the redshift of the sources studied in 
this paper, the optical depth should be $\tau \sim 1$ for photons of about $50 \mathrm{GeV}$. The highest energy photon observed is consistent with the current EBL models [7,9]. It is worth noticing that not all the sources have a hardening of the spectrum during the flaring state. For example, the gravitationally lensed blazar PKS 1830-211 at $z=2.507$, did not show any significant hardening during the huge outburst in 2010 October, while a softening of the spectrum was observed in the less-prominent flare that took place a few months after [20].

\subsection{Multi-Wavelength Variability}

The huge outburst observed in TXS 0536+145 and S5 0836+710 triggered a multi-wavelength follow up campaign to study the variability in the other bands of the electromagnetic spectrum. For TXS 0536+145 the correlated variability observed by Swift-XRT allowed the identification of the $\gamma$-ray source with the high- $z$ blazar. Before the flare no $\gamma$-ray emission was detected from TXS $0536+145$. The aim of the Swift observations was to unveil the counterpart of the $\gamma$-ray flaring object, and not a detailed study of the optical/X-ray variability. On the contrary, S5 0836+710 has been monitored by Swift for a long time. For the 2015 August flare the X-ray peak occurs a few weeks before the $\gamma$-ray peak, while for the 2015 November outburst the X-ray and $\gamma$-ray peak were simultaneous. As in $\gamma$-rays where the 2015 November flare lasted almost two weeks, also in the X-rays the flux was above its average value for several days, indicating a structured outburst in both energy bands.

The radio light curves show some variability at such long wavelengths. However, the radio variability has some time delay with respect to the variability observed at higher energies. For TXS 0536+145 the radio lags by few months, likely due to opacity effects. For both sources the radio variability is less prominent than that observed in $\gamma$-rays, where the jump in luminosity may be more than an order of magnitude. At radio frequencies the flux density increases by about a factor 2 for TXS 0536+145, while for S5 $0836+710$ the change is within a modest 20 per cent during the period monitored by our VLBA observations. However, we must notice that at the beginning of 2015 August, i.e., before the beginning of our VLBA monitoring campaign an enhancement of the radio activity was observed by the Medicina single-dish telescope [23].

\section{Conclusions}

We presented the results of multi-wavelength observations of high-redshift blazars detected by Fermi-LAT during huge $\gamma$-ray flares. Due to their high luminosity, these objects are the tip of the iceberg of the high- $z$ blazar population, with the majority too faint to be detected individually mainly for sensitivity reasons. The target sources of our investigation achieved apparent isotropic $\gamma$-ray luminosity comparable to, or even exceeding, $10^{50} \mathrm{erg} \cdot \mathrm{s}^{-1}$. The jump in luminosity is not always reflected in a hardening of the spectrum. However, during the outbursts the $\gamma$-ray spectra significantly deviate from a simple power law, and a spectral curvature is clearly present. The spectral curvature, together with the short (sub-day) time-scale variability observed in the case of PKS 2149-306 and S5 0836+710 may provide important information on the location of the flaring region and the mechanisms involved. Furthermore, multiwavelength observations will be important for shedding light on the properties of high-redshift blazars. In particular, simultaneous optical-to-X-ray observations during a $\gamma$-ray flaring activity will allow us to compare its broad-band spectral energy distribution during both low and high activity states, investigating the possible time lag, and constraining the emission mechanisms at work.

Acknowledgments: The Fermi LAT Collaboration acknowledges support from a number of agencies and institutes for both the development and the operation of the LAT as well as scientific data analysis. These include NASA and DOE in the United States, CEA/Irfu and IN2P3/CNRS in France, ASI and INFN in Italy, MEXT, KEK, and JAXA in Japan, and the K. A. Wallenberg Foundation, the Swedish Research Council and the National Space Board in Sweden. Additional support from INAF in Italy and CNES in France for science analysis during the operations phase is also gratefully acknowledged. The VLBA is operated by the US National Radio Astronomy Observatory which is a facility of the National Science Foundation operated under a cooperative agreement by Associated Universities, Inc. The European VLBI Network is a joint facility of European, Chinese, South African, and other radio astronomy institutes funded by their national research councils. The research leading to these results has 
received funding from the European Commission Seventh Framework Programme (FP/2007-2013) under grant agreement no. 283393 (RadioNet3).

Author Contributions: Monica Orienti analysed the radio and $\gamma$-ray data, and wrote the paper; Filippo D'Ammando analysed the optical, X-ray and $\gamma$-ray data, and contributed to write the paper; Marcello Giroletti performed single-dish observations and contributed to write the paper, Justin Finke contributed to discuss the results and to write the paper; Daniele Dallacasa helped in scheduling the radio observations and in the data analysis.

Conflicts of Interest: The authors declare no conflict of interest.

\section{References}

1. Acero, F.; Ackermann, M.; Ajello, M.; Albert, A.; Atwood, W.B.; Axelsson, M.; Baldini, L.; Ballet, J.; Barbiellini, G.; Bastieri, D.; et al. Fermi Large Area Telescope Third Source Catalog. Astrophys. J. 2015, 218,23

2. Baumgartner, W.H.; Tueller, J.; Markwardt, C.B.; Skinner, G.K.; Barthelmy, S.; Mushotzky, R.F.; Evans, P.A.; Gehrels, N. The 70 Month Swift-BAT All-sky Hard X-Ray Survey. Astrophys. J. 2013, 207, 19.

3. Bassani, L.; Landi, R.; Marshall, F.E.; Malizia, A.; Bazzano, A.; Bird, A.J.; Gehrels, N.; Ubertini, P.; Masetti, N. IGR J12319-0749: Evidence for another extreme blazar found with INTEGRAL. Astron. Astrophys. 2012, 543, A1.

4. Ghisellini, G.; Sbarrato, T.; Tagliaferri, G.; Foschini, L.; Tavecchio, F.; Ghirlanda, G.; Braito, V.; Gehrels, N. SDSS J114657.79+403708.6: The third most distant blazar at $\mathrm{z}=5.0$. Mon. Not. R. Astron. Soc. 2014, 440, L111-L115.

5. Sbarrato, T.; Tagliaferri, G.; Ghisellini, G.; Perri, M.; Puccetti, S.; Baloković, M.; Nardini, M.; Stern, D.; Boggs, S.E.; Brandt, W.N.; et al. NuSTAR Detection of the Blazar B2 1023+25 at Redshift 5.3. Astrophys. J. 2013, 777, 147.

6. Ghisellini, G.; Tagliaferri, G.; Foschini, L.; Ghirlanda, G.; Tavecchio, F.; Della Ceca, R.; Haardt, F.; Volonteri, M.; Gehrels, N. High-redshift Fermi blazars. Mon. Not. R. Astron. Soc. 2011, 411, 901-914.

7. D'Ammando, F.; Orienti, M. High-energy properties of the high-redshift flat spectrum radio quasar PKS 2149-306. Mon. Not. R. Astron. Soc. 2016, 455, 1881-1891.

8. Ackermann, M.; Ajello, M.; Allafort, A.; Atwood, W.B.; Baldini, L.; Ballet, J.; Barbiellini, G.; Bastieri, D.; Bechtol, K.; Belfiore, A.; et al. The First Fermi-LAT Catalog of Sources above $10 \mathrm{GeV}$. Astrophys. J. Suppl. Ser. 2013, 209, 34 .

9. Orienti, M.; D'Ammando, F.; Giroletti, M.; Finke, J.; Ajello, M.; Dallacasa, D.; Venturi, T. Exploring the multiband emission of TXS 0536+145: The most distant $\gamma$-ray flaring blazar. Mon. Not. R. Astron. Soc. 2014, $444,3040-3051$.

10. Sowards-Emmerd, D.; Romani, R.W.; Michelson, P.F.; Healey, S.E.; Nolan, P.L. A Northern Survey of Gamma-Ray Blazar Candidates. Astrophys. J. 2005, 626, 95-103.

11. Abdo, A.A.; Ackermann, M.; Ajello, M.; Allafort, A.; Antolini, E.; Atwood, W.B.; Axelsson, M.; Baldini, L.; Ballet, J.; Barbiellini, G.; et al. Fermi Large Area Telescope First Source Catalog. Astrophys. J. Suppl. Ser. 2010, 188, 405-436.

12. Nolan, P.L.; Abdo, A.A.; Ackermann, M.; Ajello, M.; Allafort, A.; Antolini, E.; Atwood, W.B.; Axelsson, M.; Baldini, L.; Ballet, J.; et al. Fermi Large Area Telescope Second Source Catalog. Astrophys. J. Suppl. Ser. 2012, 199, 31.

13. Tagliaferri, G.; Ghisellini, G.; Perri, M.; Hayashida, M.; Balok', M.; Covino, S.; Giommi, P.; Madejski, G.M.; Puccetti, S.; Sbarrato, T.; et al. NuSTAR and Multifrequency Study of the Two High-redshift Blazars S5 0836+710 and PKS 2149-306. Astrophys. J. 2015, 807, 167.

14. Thompson, D.J.; Bertsch, D.L.; Dingus, B.L.; Fichtel, C.E.; Hartman, R.C.; Hunter, S.D.; Kanbach, G.; Kniffen, D.A.; Lin, Y.C.; Mattox, J.R.; et al. EGRET observations of active galactic nuclei. Astrophys. J. 1993, 415, L13-L16.

15. Abdo, A.A.; Ackermann, M.; Ajello, M.; Atwood, W.B.; Axelsson, M.; Baldini, L.; Ballet, J.; Band, D.L.; Barbiellini, G.; Bastieri, D.; et al. Fermi/Large Area Telescope Bright Gamma-Ray Source List. Astrophys. J. Suppl. Ser. 2009, 183, 46-66. 
16. Akyuz, A.; Thompson, D.J.; Donato, D.; Perkins, J.S.; Fuhrmann, L.; Angelakis, E.; Zensus, J.A.; Larsson, S.; Sokolovsky, K.; Kurtanidze, O. Long-term multiwavelength studies of high-redshift blazar 0836+710. Astron. Astrophys. 2013, 556, A71.

17. Abdo, A.A.; Ackermann, M.; Ajello, M.; Allafort, A.; Baldini, L.; Ballet, J.; Barbiellini, G.; Bastieri, D.; Bellazzini, R.; Berenji, B.; et al. Fermi Gamma-ray Space Telescope Observations of the Gamma-ray Outburst from 3C454.3 in November 2010. Astrophys. J. 2011, 733, L26.

18. Ackermann, M.; Ajello, M.; Atwood, W.B.; Baldini, L.; Ballet, J.; Barbiellini, G.; Bastieri, D.; Becerra Gonzalez, J.; Bellazzini, R.; Bissaldi, E.; et al. The Third Catalog of Active Galactic Nuclei Detected by the Fermi Large Area Telescope. Astrophys. J. 2015, 810, 14.

19. Abdo, A.A.; Ackermann, M.; Ajello, M.; Antolini, E.; Baldini, L.; Ballet, J.; Barbiellini, G.; Bastieri, D.; Baughman, B.M.; Bechtol, K.; et al. The Fermi-LAT High-Latitude Survey: Source Count Distributions and the Origin of the Extragalactic Diffuse Background. Astrophys. J. 2010, 720, 435-453.

20. Abdo, A.A.; Ackermann, M.; Ajello, M.; Allafort, A.; Amin, M.A.; Baldini, L.; Barbiellini, G.; Bastieri, D.; Bechtol, K.; Bellazzini, R.; et al. Gamma-Ray Flaring Activity from the Gravitationally Lensed Blazar PKS 1830-211 Observed by Fermi LAT. Astrophys. J. 2015, 799, 143.

21. Franceschini, A.; Rodighiero, G.; Vaccari, M. Extragalactic optical-infrared background radiation, its time evolution and the cosmic photon-photon opacity. Astron. Astrophys. 2008, 487, 837-852.

22. Finke, J.D.; Razzaque, S.; Dermer, C.D. Modeling the Extragalactic Background Light from Stars and Dust. Astrophys. J. 2010, 712, 238-249.

23. Giroletti, M.; Righini, S.; Bach, U.; D’Ammando, F.; Orienti, M.; Raiteri, C.M.; Villata, M. Multi-frequency radio observations of S5 0836+710 after gamma-ray outburst. Astron. Telegr. 2015, 7890. Available online: http:/ / www.astronomerstelegram.org/?read=7890 (accessed on 7 August 2015).

(C) 2016 by the authors; licensee MDPI, Basel, Switzerland. This article is an open access article distributed under the terms and conditions of the Creative Commons Attribution (CC-BY) license (http:/ / creativecommons.org/licenses/by/4.0/). 